\title{
A monolithic 12-bit digitally calibrated D/A converter
}

\author{
Hong Liu ${ }^{1,2}$, Le $\mathrm{Xu}^{1,2}$, and Tong Tian ${ }^{1, \text { a) }}$
}

\begin{abstract}
A monolithic 12-bit digitally calibrated digital-to-analog converter (DAC) has been developed. The principle of proportional match design in the resistor switch network, its structural characteristics and the resistor network for calibration are presented. A close-loop calibration circuit with differential difference amplifier (DDA) has been proposed to eliminate the variation of switch resistance caused by temperature and supply-voltage. Moreover, this paper also presents the proposed automatic calibration algorithm based on fuse trim, which can reduce the number of times to trim the resistors and simplify the trimming process. The proposed self-calibrated and digitally trimmed DAC has been fabricated with a $0.5 \mu \mathrm{m}$ bipolar-CMOS-DMOS (BCDMOS) process. Measurement results show that the integral nonlinearity (INL) and differential nonlinearity (DNL) of the developed DAC after calibration achieve -0.65 LSB +0.6 LSB and $-0.6 \mathrm{LSB} \sim+0.25 \mathrm{LSB}$, respectively. The settling time of the developed DAC achieves $1 \mu \mathrm{s}$.

Keywords: R-2R, voltage-mode, digital-to-analog converter, automatic calibration algorithm, differential difference amplifier

Classification: Integrated circuits (memory, logic, analog, RF, sensor)
\end{abstract}

\section{Introduction}

Up until recently, high-resolution digital-to-analog converters (DACs) are widely used in industrial process control applications such as programmable logic controllers and sensor controllers $[1,2,3,4,5,6,7,8,9,10,11,12,13]$. In these applications, the DAC is part of a sensor/controller feedback system and used for controlling such devices as motors and cylinders based on the sensors inputs. Therefore, dynamic key performances indicators such as spurious-free dynamic range (SFDR) and signal-to-noise-and-distortion ratio (SNDR) are not subject to strict requirements; however, high resolution and fast settling time to control the objects accurately and fast are more critical $[1,2]$. For example, in the application of automatic control equipment, the accuracy of DAC is usually required to be 12 bit 16 bit, and the settling time is required to be $1 \mu \mathrm{s} \sim 100 \mu \mathrm{s}$.

For implementing this type of DAC, the R-2R mode is a preferred choice because of its simple structure, area efficiency and low cost $[14,15]$. In traditional $N$-bit voltagemode R-2R ladder DAC shown in Fig. 1 ( $N$ is the resolution of the DAC), two issues should be taken into consideration in order to obtain high resolution. Firstly, the accuracy of the DAC depends on the matching tolerance of the resistors

${ }^{1}$ Shanghai Institute of Microsystem and Information Technology, Chinese Academy of Sciences, Shanghai, China

${ }^{2}$ University of Chinese Academy of Sciences, Beijing, China

a) tiantong@mail.sim.ac.cn

DOI: 10.1587/elex.17.20200371

Received November 2, 2020

Accepted December 14, 2020

Publicized December 22, 2020

Copyedited January 25, 2021 that constitute the ladder; 8 10-bit DACs have been common practice in existing processes with careful layout. For 12-bit resolution or above, some designs choose the segmented R-2R ladder architecture [16], and several studies focusing on improving the linearity have been carried out. The laser trimming technique [17] using thin/thick film process can improve the linearity effectively, but it needs extra manufacturing layers and large area for trimming, which increases the cost. The ordered element matching (OEM) technique $[18,19,20,21]$ and the dynamic element matching (DEM) technique $[22,23,24]$ can also reduce the mismatch effects. Secondly, another main source of error in the R-2R ladder D/A converter is the mismatch in on-resistance of the switches. Several solutions $[25,26]$ have been proposed to equalize resistance of switches connected to the reference voltage and ground.

As for the trimming procedure, the calibration algorithm of the traditional R-2R DAC needs to record the output values, so as to extract the resistance weight of each bit, and then calculate the trimming code. Since trimming one bit of the DAC will affect the weight of other bits, trimming is done for each bit from the LSB to the MSB $[16,17]$. The procedure needs to trim the resistors for many times, which is complicated and not efficient.

In contrast to the above works, in this paper, a novel circuit is proposed to calibrate the switches resistance. Moreover, we propose a digitally calibration algorithm based on fuse trim, with the aim to reduce the number of times to trim the resistors and simplify the trimming process. This proposed calibration circuit of the switches resistance and digital calibration algorithm are implemented on a monolithic 12-bit CMOS DAC using a 5-7 segmented R-2R ladder architecture. The remainder of the paper is organized as follows. Section 2 explains the system architecture and circuit designs. Section 3 details the automatic calibration algorithm of the DAC. The measurement results are presented in Section 4. Finally, Section 5 draws the conclusion.

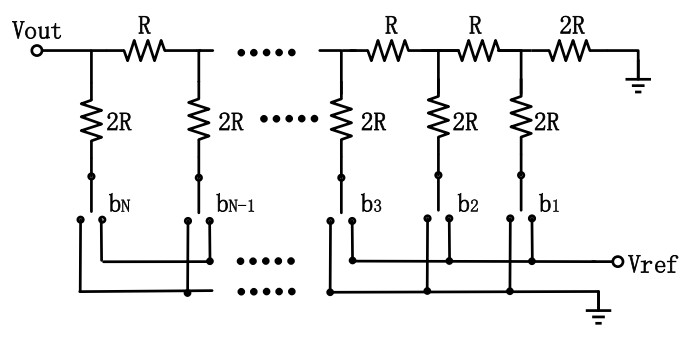

Fig. 1 Traditional voltage-mode DAC structure. 


\section{System architecture and circuit design}

\subsection{System architecture}

In this paper, the proposed DAC adopts a voltage-type R$2 \mathrm{R}$ ladder network. Limited by the variation of the $0.5 \mu \mathrm{m}$ BCDMOS process from CSMC, the mismatch precision of resistors can achieve $0.2 \%$ when the width and length are $20 \mu \mathrm{m}$ and $100 \mu \mathrm{m}$, respectively.

Considering the impact of temperature and voltage change, the final comparative match precision of resistors can be regarded to be $0.5 \%$. In comparison, conventional R-2R networks can ensure a DAC accuracy as high as 8bit, while trimming is capable of increasing the accuracy further [27].

The proposed DAC adopts a segmented resistor array, as depicted in Fig. 2. $b 1 \sim b 12$ represent the switches of bit 1 to bit 12 , respectively. The segmented R-2R DAC consists of 5-bit binary array and 7-bit R-2R array. The former part is the most significant bit (MSB) array, whereas the other part is the least significant bit (LSB) array. Therefore, we can trim the 5-bit MSB array while maintaining the accuracy of the 7-bit LSB array, obtaining a 12-bit accuracy. Since the variation of each resistor has modest impact on the adjacent bits, all the bits can be calibrated at the same time, which thereby increase the efficiency of trimming to a great extent. The five MSBs adopt five resistor trimming blocks which are controlled by six bits $D<5: 0>$, as shown in Fig. 2 .

In order to achieve 12-bit accuracy, the resistors in the network should satisfy a match accuracy of $1 / 4096$. At the same time, the turn-on resistors and trimming resistors are also supposed to satisfy the accuracy of $1 / 4096$. We define the turn-on resistors of switches $b_{1} \sim b_{12}$ as $R_{1} \sim R_{12}$, respectively. Taking the LSB array as an example, the resistor network should satisfy the following equation:

$$
R+R+1 / 2 R_{1}=2 R+R_{2}
$$

Then $R_{1}=2 R_{2}$. Based on the similar approach, the following relationship can be obtained:

$$
R_{1}=2 R_{2}=4 R_{3}=\cdots=2^{11} R_{12}
$$

Next, we define the dimension of the switch MOSFETs of each bit as $(W / L)_{1},(W / L)_{2}, \ldots,(W / L)_{12}$, respectively. In

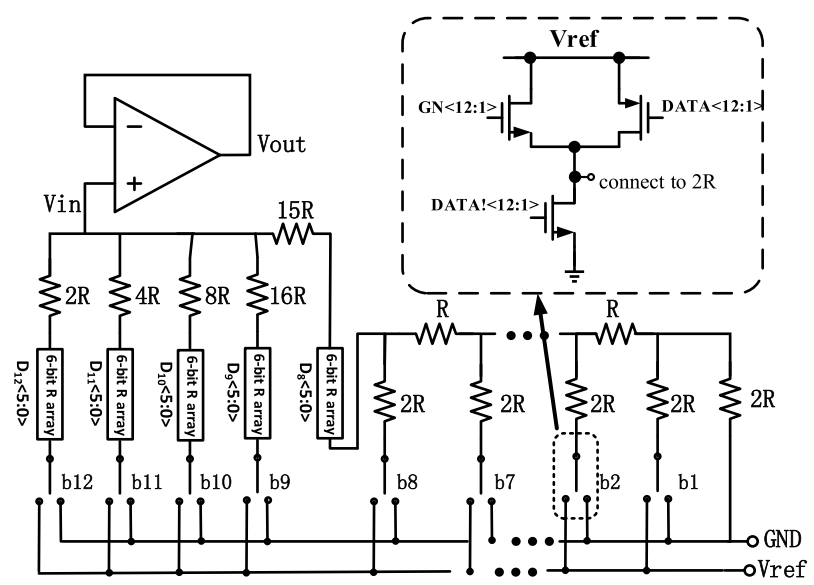

Fig. 2 The schematic of the proposed DAC that builds upon a segmented resistor array, splitting the 4MSBs from the R-2R network. order to ensure that the drain voltages and source voltages of these MOSFETs are identical, their dimension should satisfy the following relationship:

$$
(W / L)_{1}=1 / 2(W / L)_{2}=1 / 4(W / L)_{3}=\cdots=1 / 2^{11}(W / L)_{12}
$$

Nevertheless, the resistance of switch MOSFETs may change with the variation of temperature and supply voltage when these MOSFETs connect Vref and GND, which will degrade the precision of the DAC. A topology to alleviate this impact is described in the following.

\subsection{Switches calibration circuit}

From Section 2.1, it is known that the switch resistances should be equal when the switches connect to Vref and $G N D$. In practice, due to the variations of temperature and voltage, the switches' resistances match badly. In our DAC, a calibration method is proposed to decrease the mismatch of switch resistors due to the above variations.

The calibration circuit is shown in Fig. 3. M12 and M13 represent the switches which connect to Vref, M14 represents the switches which connect to $G N D$, and $R$ represents the ladder resistors in the network. The switch network is controlled by three voltages: VDD, GND and $G N(<V D D)$. The amplifier included in Fig. 3 uses a modified structure of the differential difference amplifier (DDA) [28, 29]. The output of the DDA connects to the gate of M12, which forms a negative feedback. This circuit makes the differential input voltages of the DDA become equal.

$$
\begin{gathered}
V_{r e f}-V 1=V 2-V_{G N D} \\
I=\frac{V_{r e f}-V 1}{R_{M 12|| M 13}}=\frac{V 2-V_{G N D}}{R_{M 14}}
\end{gathered}
$$

According to eqs. (4) and (5), $R_{M 12|| M 13}=R_{M 14}$ can be obtained, where $R_{M 12|| M 13}$ is the switch resistance of M12 in parallel with M13, $R_{M 14}$ is the switch resistance of M14, and $I$ is the current flowing through resistor $R$.

As shown in Fig. 3, the DDA consists of two stages, a differential-input single-ended output transconductance stage and a common-source gain stage. The DDA uses

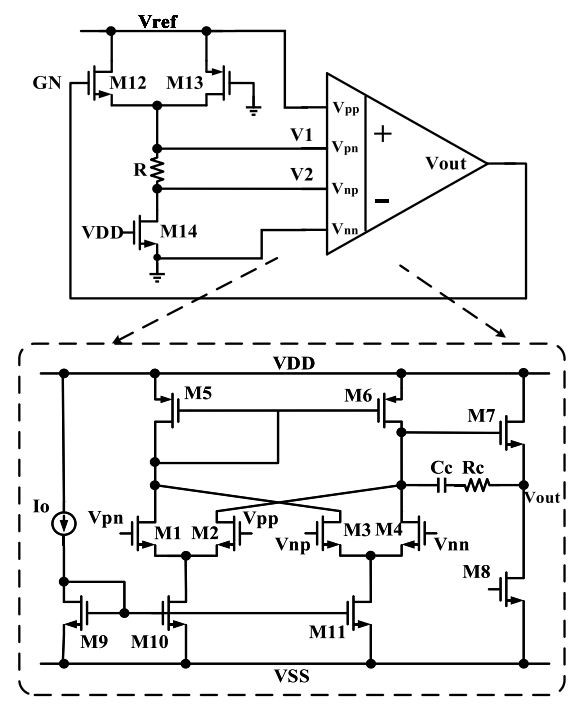

Fig. 3 The schematic of the proposed calibration circuit and DDA. 
two differential pairs (M1, M2, M3, M4) to realize the four input ports. A compensating capacitor $(C c)$ and a resistor $(R c)$ are employed to ensure stability. The values of $C c$ and $R c$ are $5 \mathrm{pF}$ and $4.8 \mathrm{k} \Omega$ respectively. The open-loop gain of the DDA is up to $60 \mathrm{~dB}$ to ensure the accuracy of switch resistance.

\subsection{Fuse resistor network}

The fuse calibration network of the DAC is shown in Fig. 4. The calibration network is designed based on the trim fuse. The trim resistance value of each bit should keep the ratio of $2^{\mathrm{N}}$. The two ports of resistor $R$ cal are connected to the trim pads. When the selective signals $D<5: 0>$ are asserted, the switches of the selected bits turn on, and then the selected fuses will be blown.

Since the total variation range of the network resistors is $\pm 0.5 \%$, the trimming capability of the network should cover this range and hence is set to be $\pm 0.7 \%$, leaving some margins. Meanwhile, the trimming precision of each bit should reach $1 / 4096$ of the corresponding resistance, so as to ensure the total trimming precision within $1 \mathrm{LSB}$. Then approximate calculation reveals that a 58-step precision is a necessary, which requires 6 bits. Take the trimming resistor of the $12^{\text {th }}$ bit depicted in the Fig. 4 as an example. Due to the large trimming range and low precision, the mode of series resistance will be difficult to achieve, so the trimming network adopts the parallel mode. The parallel mode can also reduce the number of PAD cells for trimming, thus greatly reducing the area of the chip. Because the main resistor $2 \mathrm{R}$ of the $12^{\text {th }}$ bit is $12 \mathrm{k} \Omega$, the lowest precision is $2.93 \Omega$. If we define the trim resistor $R$ cal of $12^{\text {th }}$ bit as $450 \Omega$ and $R \mathrm{x}$ as $1.2 \mathrm{k} \Omega$, it can be conclude that the trim accuracy is between $1.8 \Omega$ and $2.6 \Omega$, and the trim range is $176 \Omega(36 \mathrm{LSB})$.

Bit 10, bit 11 and bit 12 adopt the trimming circuit described in Fig. 4(a). However, with the resistor $R$ cal and $R \mathrm{x}$ increasing in the proportion of $2^{\mathrm{N}}$, the value of the trimming resistors becomes unacceptable. Therefore, the calibration circuits of bit 8 and bit 9 adopt segmented networks, as depicted in Fig. 4(b). By reusing the resistor Rcal in the signal path, the trimming resistors value can be reduced by half.

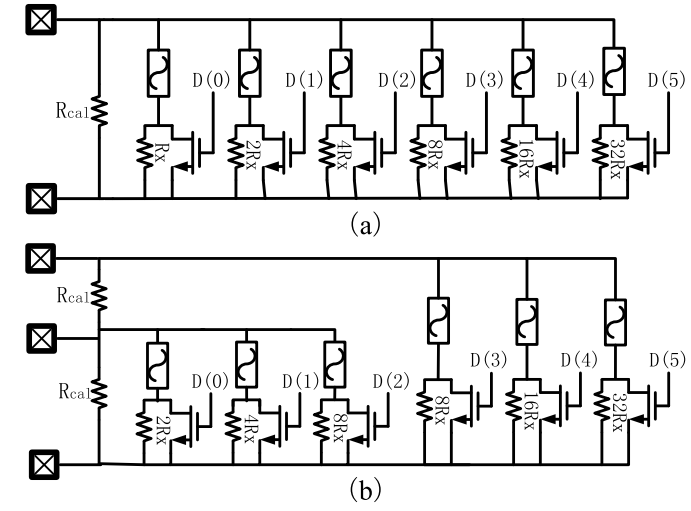

Fig. 4 The schematic of (a) binary fuse resistor network, (b) segment fuse resistor network.

\section{Automatic calibration algorithm}

A new automatic calibration algorithm is proposed to reduce the number of times to trim the resistors, and simplify the trimming process. It is well known that the accuracy of $\mathrm{R}-2 \mathrm{R}$ DAC is mainly decided by the matching level of the resistors in the MSB array. So, the purpose of trimming is to make the resistance value of each MSB stage into the ratio of $2^{\mathrm{N}}$. Firstly, it is necessary to calculate the relative value of the resistance at each stage from the output voltage. The equivalent structure of the MSB array is shown in Fig. 5. $V_{\mathrm{o}}$ is the output voltage of DAC. When the switch of bit $\mathrm{i}$ connects to Vref and the switches of the other bits connect to $G N D$, the output voltage can be expressed as:

$$
V_{o, i}=\frac{R_{o, i}}{R_{o, i}+R_{i}} V_{r e f}=\frac{R_{j} \operatorname{Re}(i, j)}{R_{j} \operatorname{Re}(i, j)+R_{j} R_{i}+R_{i} \operatorname{Re}(i, j)} V_{r e f}
$$

where, $R_{\mathrm{i}}$ and $R_{\mathrm{j}}$ are the resistor value of bit $\mathrm{i}$ and bit $\mathrm{j}, R_{\mathrm{o}, \mathrm{i}}$ is the equivalent resistor value of the other stages except bit $i$, and $\operatorname{Re}(i, j)$ is the equivalent resistor value of the other stages except bit $i$ and bit $j$. We normalize the output voltage of the bit $i$ as:

$$
\alpha_{i}=\frac{2^{i} V_{o, i}}{V_{r e f}}-1
$$

$\alpha_{j}$ can also be obtained. Suppose that $\alpha_{\mathrm{i}}=\alpha_{j}$, then

$$
\frac{R_{\mathrm{i}}}{2^{i}}=\frac{R_{j}}{2^{\mathrm{j}}}
$$

It is known from eq. (8) that, when the normalization coefficient $\alpha$ of each stage is equal, the resistance value of each stage will be the ratio of $2^{\mathrm{N}}$.

Assume that the error of the $\mathrm{i}^{\text {th }}$ bit resistor is $\beta_{\mathrm{i}}$. The output resistance $R_{\text {out }}$ of the network is

$$
R_{\text {out }}=1 /\left(\sum_{i=1}^{N} \frac{1}{2^{i} R\left(1+\beta_{i}\right)}\right)
$$

The contribution of the $\mathrm{i}^{\text {th }}$ bit to the output voltage is

$$
V_{o, i}=\frac{R_{\text {out }} V_{\text {ref }}}{2^{i} R\left(1+\beta_{i}\right)}
$$

When the $\mathrm{k}^{\text {th }}$ bit is turned on individually, the normalization coefficient $\alpha_{i}$ of the $\mathrm{i}^{\text {th }}$ bit of the resistance network is

$$
\alpha_{i}=\frac{2^{i} V_{o, i}}{V_{r e f}}-1=\frac{1}{R\left(1+\beta_{i}\right) \sum_{k=1}^{\mathrm{N}}\left(\frac{1}{2^{k} R\left(1+\beta_{k}\right)}\right)}-1
$$

Assuming that $\mathrm{N}=3, \beta_{2}=0, \beta_{3}=0$, according to eq. (11) we get the normalization coefficient when each bit

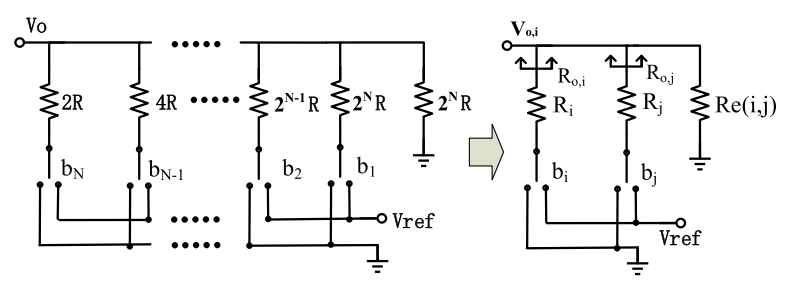

Fig. 5 Equivalent structure of the MSB array. 
is turned on individually. The normalization coefficient is $\alpha_{1}=-\alpha_{2}=-\alpha_{3}=-\beta_{1} /\left(2+\beta_{1}\right)$. It can be concluded that the normalization value of the trimming bit has similar magnitude and opposite direction to the other bits. So, trimming one bit will not affect all other bits.

Based on the above conclusion, we propose an automatic fuse trimming algorithm whose procedure is shown in Fig. 6. Because the resistance value of each bit is proportional to the output voltage when each bit is turned on individually, we can obtain the resistance proportion of each bit by measuring the output voltage when each bit is turned on individually. Then we choose the minimum value of the output voltage of the five high bits, and calculate the relative normalization coefficient $\alpha$ between the minimum value and the other four bits. Through blowing the appropriate fuse, the resistance ratio of the five high bit will back to $2^{\mathrm{N}}$.

The detailed flow chart of automatic fuse trimming is shown in Fig. 7. Firstly, we record the output voltages of the DAC when switches of $b 1-b 12$ are turned on, individually. The output voltages of the DAC are defined as Vout 1 -Vout 12. If the errors of Vout 1 -Vout 7 are within \pm 0.5 LSB and the errors of Vout 8 -Vout 12 within the trimming range of $\pm 0.7 \%$, the trimming procedure goes to the next step. Secondly, use

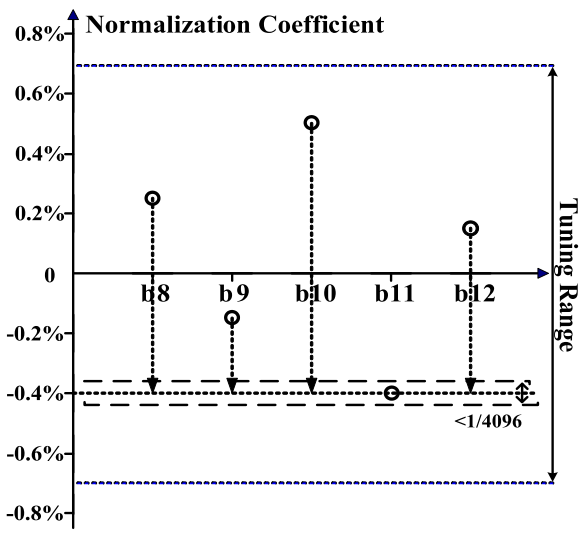

Fig. 6 The diagram of fuse trimming procedure.

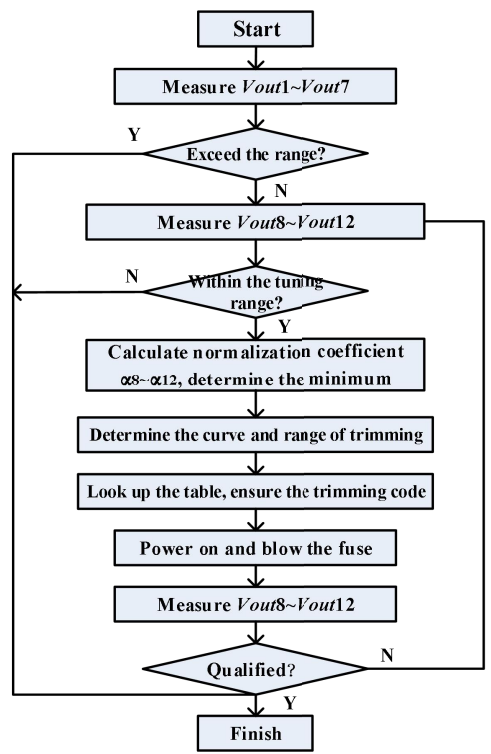

Fig. 7 The flow chart of digitally calibration algorithm. the eq. (7) to normalize the output voltage Vout8-Vout 12 and get the normalization minimum value. Thirdly, choose the normalization minimum value of the output voltage Vout8Vout 12 as the base point, then move the other four points towards the base point and calculate the relative values between the minimum value and the other four points as is shown in Fig. 6. Fourthly, according to the look-up table of trimming stored in the tester, the trimming codes of bit 8 to bit 12 are selected. Fifthly, according to the six bit trimming codes, blow the corresponding fuses from bit 12 to bit 8 .

This algorithm can be extended to trim DACs with arbitrarily accuracy which adopts this structure. It can be applied to the situations where the fuse resistors can only increase or decrease, as well as change bilaterally.

In the paper, "trimming times", which is defined as the number of times to trim the resistor, is used to evaluate the trimming methods. For the traditional trim of R-2R structure, calibration is done bottom-up, starting from the LSB to the MSB, trimming one bit will affect the weight of all other bits. So the procedure needs to trim the resistors for many times. For Ref. [17] and [30], the "trimming times" are $N+4$ and $N$, respectively. The "trimming time" of the proposed circuit is only 1 , which can reduce the number of times to trim the resistors and simplify the trimming process.

\section{Chip measurement results}

The 4-channel 12-bit DAC has been fabricated with a 0.5$\mu \mathrm{m}$ BCDMOS process from CSMC. Fig. 8 shows the die photograph of the DAC. The whole area of the DAC is $12.74 \mathrm{~mm}^{2}$ including PADs and seal ring. This area includes the resistor array, input switches, trim circuits, DDA circuits, output buffers and digital logic. The area of the trim circuit occupies $16 \%$ of the whole chip.

The static power consumption is calculated with the measured current from the supply voltage in the proposed circuit. The 4-channel DAC consumes $128 \mathrm{~mW}$ from a $15 \mathrm{~V}$ power supply. The average power consumption of one channel is $32 \mathrm{~mW}$. Since the load capacitance of the output buffer is $100 \mathrm{pF}$, in order to achieve a fast voltage transition, the output buffer consumes $24 \mathrm{~mW}$ ( $75 \%$ of the total power consumption). An external reference of $10 \mathrm{~V}$ is used. The DAC test and trimming are done on wafer, with the help of a Teradyne J750 ATE tester. Firstly, we measure the output voltage values of the DAC when the switches of $b 1 \sim b 12$ are turned on individually, using the parameter measurement units (PMU) of the J750 ATE tester. Then we store the data into the memory. In order to eliminate the impact of environmental interference, each group of data is measured twice and

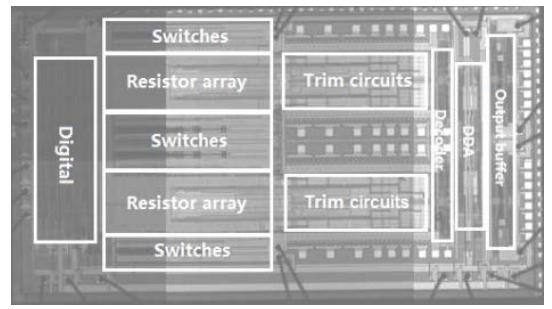

Fig. 8 Die micrograph of the DAC. 


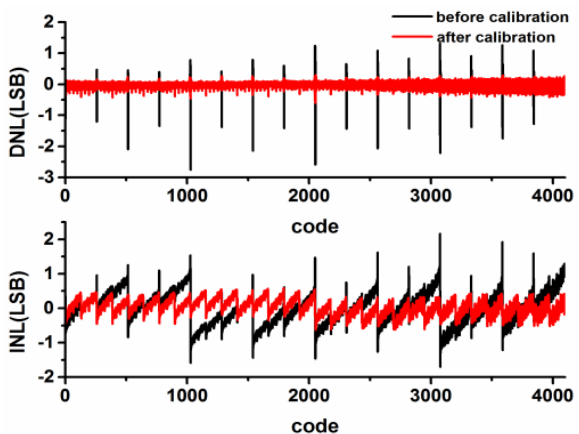

Fig. 9 INL/DNL measurement results before and after calibration.

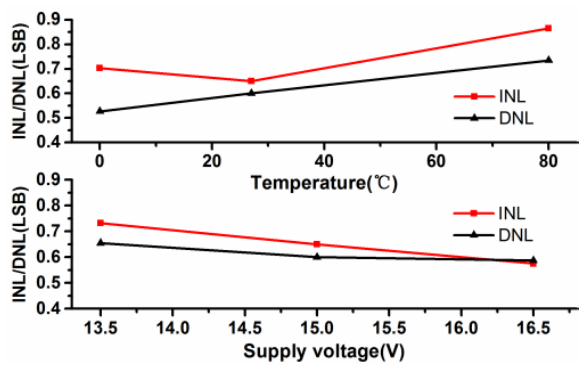

Fig. 10 INL/DNL measurement results over temperature and supply voltage.

the average value is used. After that, the tester calls the automatic calibration algorithm program to burn the corresponding fuse according to the calculated trim code. Then the DAC calibration is completed, which greatly improves the efficiency of the trimming. Fig. 9 shows the performance of INL/DNL before and after calibration. The measured INL and DNL before calibration are -1.9 LSB +2.2 LSB and $-2.8 \mathrm{LSB}+1.4 \mathrm{LSB}$, respectively. The measured INL and DNL after calibration are $-0.65 \mathrm{LSB} \sim+0.6 \mathrm{LSB}$ and $-0.6 \mathrm{LSB} \sim+0.25 \mathrm{LSB}$, respectively. Measurement results show that the automatic calibration algorithm has a significant effect on the static performance of the developed DAC. INL and DNL are tested after calibration at various temperatures $\left(0^{\circ} \mathrm{C}, 27^{\circ} \mathrm{C}\right.$ and $\left.80^{\circ} \mathrm{C}\right)$ and various supply-voltages $(13.5 \mathrm{~V}, 15 \mathrm{~V}$ and $16.5 \mathrm{~V})$. The test results are shown in Fig. 10. The maximum values of the tested INL and DNL are both within 1 LSB. The results demonstrate that the developed DAC with the switch calibration circuit is little affected by temperature and supply-voltage variations. When measuring the settling time, the input digital code is changed from all " 0 " to all " 1 ", and the time for the output voltage to reach $\pm 1 / 2$ LSB of full scale amplitude is recorded. This process is completed by the J750 ATE tester. The settling time of the developed DAC is $1 \mu \mathrm{s}$.

Table I summaries the achieved performance of the developed DAC, and compares it with other state-of-the-art DACs. The INL and DNL of all works are within \pm 1 LSB. Compared with Ref. [17] and [31], this paper reduces the number of times to trim resistors and simplifies the trimming process. The current consumption measured in this paper is equivalent to that of Ref. [31], and smaller than that of Ref. [30]. Ref. [17] does not describe the load of the output buffer, and the power supply voltage is $3.3 \mathrm{~V}$, so the power consumption cannot be compared directly.
Table I Comparison with the state-of-the-art works.

\begin{tabular}{c|c|c|c|c}
\hline & {$[17]$} & {$[30]$} & {$[31]$} & This Work \\
\hline Process $(\mu \mathrm{m})$ & 0.25 & 0.5 & NA & 0.5 \\
\hline Number of Channels & 1 & 4 & 4 & 4 \\
\hline Area (mm²) & $\begin{array}{c}0.47(\mathrm{core} \\
\text { area) }\end{array}$ & 49 & NA & 12.74 \\
\hline Calibration Method & laser-trim & NA & laser-trim & fuse-trim \\
\hline Calibration Area overhead & $4.4 \%$ & NA & $>30 \%$ & $16 \%$ \\
\hline Trimming Times** & $N+4$ & NA & $N$ & 1 \\
\hline INL/DNL (LSB) & $\pm 0.7 / \pm 0.2$ & $\pm 0.67 / \pm 0.23$ & $\pm 1 / \pm 1$ & $\pm 0.65 / \pm 0.6$ \\
\hline Supply voltage (V) & 3.3 & \pm 15 & \pm 15 & 15 \\
\hline Settling Time ( $\mu \mathrm{s})$ & 1 & 10 & 10 & 1 \\
\hline Reference Input Range (V) & 3.3 & 10 & 10 & 10 \\
\hline Power Consumption (mW) & 1.7 & $131^{*}$ & $62.5^{*}$ & $32^{*}$ \\
\hline Resolution & 14 -bit & 12 -bit & 12 -bit & 12 -bit \\
\hline
\end{tabular}

* Power consumption of one channel (include buffer) $@ C_{L}=100 \mathrm{pF}$.

** Defined as the number of times to trim the resistors.

\section{Conclusion}

In this paper, a monolithic 12-bit D/A converter has been developed. In order to eliminate the variation of switch resistance value caused by temperature and supply voltage, a new calibration circuit has been proposed. Moreover, this paper proposed an automatic calibration algorithm based on fuse trim, which can reduce the number of times to trim the resistors. The proposed self-calibrated and digitally trimmed DAC has been fabricated with a $0.5 \mu \mathrm{m}$ BCDMOS process. Test results show that the INL and DNL of the developed DAC after calibration are -0.65 LSB +0.6 LSB and $-0.6 \mathrm{LSB}+0.25 \mathrm{LSB}$, respectively, and the settling time of the developed DAC is $1 \mu \mathrm{s}$.

\section{References}

[1] R.C. McLachlan, et al.: "A 20b clockless DAC with sub-ppm INL $7.5 \mathrm{nV} / \sqrt{\mathrm{Hz}}$ noise and $0.05 \mathrm{ppm} /{ }^{\circ} \mathrm{C}$-stability," IEEE J. Solid-State Circuits 48 (2013) 3028 (DOI: 10.1109/JSSC.2013.2278449).

[2] W. Guo, et al.: "An area- and power-efficient $I_{\text {ref }}$ compensation technique for voltage mode $R-2 R$ DACs," IEEE Trans. Circuits Syst. II, Exp. Briefs 62 (2015) 656 (DOI: 10.1109/TCSII.2015.2406351).

[3] A.A. Noorwali, et al.: "A 16-bit 4 MSPS DAC for lock-in amplifier in 65nm CMOS," IEEE 13th International Conference on Networking, Sensing, and Control (2016) 1 (DOI: 10.1109/ICNSC.2016. 7478965).

[4] A. Esmaili and H. Babazadeh: "A robust calibration method for R-2R ladder-based current-steering DAC," AEU-International Journal of Electronics and Communications 111 (2019) 152887 (DOI: 10.1016/J.AEUE.2019.152887).

[5] K. Hokazono, et al.: "A novel high-precision DAC utilizing tribonacci series," IEICE Electron. Express 9 (2012) 515 (DOI: 10.1587/ elex.9.515)

[6] J. Yuan, et al.: "A resistance matching based self-testable currentmode R-2R digital-to-analog converter," IEICE Electron. Express 10 (2013) 20130753 (DOI: 10.1587/elex.10.20130753).

[7] H.-C. Seol, et al.: "An area-efficient high-resolution resistor-string DAC with reverse ordering scheme for active matrix flat-panel display data driver ICs," J. Display Technol. 12 (2016) 828 (DOI: 10.1109/ JDT.2016.2526042). 
[8] Y. Cong and R.L. Geiger: "A 1.5-V 14-bit 100-MS/s self-calibrated DAC," IEEE J. Solid-State Circuits 38 (2003) 2051 (DOI: 10.1109/ JSSC.2003.819163).

[9] F.-T. Chou, et al.: "A compact 12-bit DAC with novel bias scheme," IEICE Electron. Express 11 (2014) 20140572 (DOI: 10.1587/elex.11. 20140572).

[10] Q. Huang and F. Yu: "A 10-bit 0.41-mW 3-MSps R-I DAC with fullswing output voltage," IEICE Electron. Express 15 (2018) 20180377 (DOI: 10.1587/elex.15.20180377).

[11] T. Miki, et al.: "An 80-MHz 8-bit CMOS D/A converter," IEEE J. Solid-State Circuits 21 (1986) 983 (DOI: 10.1109/JSSC.1986. 1052639).

[12] B. Ma, et al.: "A 12-bit 1.74-mW 20-MS/s DAC with resistor-string and current-steering hybrid architecture," System-on-Chip Conference (2015) 1 (DOI: 10.1109/SOCC.2015.7406897).

[13] H. Klimach, et al.: "An M-2M digital-to-analog converter design methodology based on a physical mismatch model," IEEE International Symposium on Circuits and Systems (2008) 2254 (DOI: 10.1109/ISCAS.2008.4541902).

[14] T.-C. Lee and C.-H. Lin: "Nonlinear R-2R transistor-only DAC," IEEE Trans. Circuits Syst. I, Reg. Papers 57 (2010) 2644 (DOI: 10.1109/TCSI.2010.2046973).

[15] D. Marche and Y. Savaria: "Modeling $R-2 R$ segmented-ladder DACs," IEEE Trans. Circuits Syst. I, Reg. Papers 57 (2010) 31 (DOI: 10.1109/TCSI.2009.2019396)

[16] J.R. Naylor: "A complete high-speed voltage output 16-bit monolithic DAC," IEEE J. Solid-State Circuits 18 (1983) 729 (DOI: 10.1109/ JSSC.1983.1052024).

[17] D. Marche, et al.: "Laser fine-tuneable deep-submicron CMOS 14bit DAC," IEEE Trans. Circuits Syst. I, Reg. Papers 55 (2008) 2157 (DOI: 10.1109/TCSI.2008.920152).

[18] T. Zeng and D. Chen: "An order-statistics based matching strategy for circuit components in data converters," IEEE Trans. Circuits Syst. I, Reg. Papers 60 (2013) 11 (DOI: 10.1109/TCSI.2012.2232991).

[19] Y. Li and D. Chen: "Low-cost, high-precision DAC design based on ordered element matching," IEEE Trans. Circuits Syst. I, Reg. Papers 66 (2019) 502 (DOI: 10.1109/TCSI.2018.2870665).

[20] Y. Li and D. Chen: "A novel 20-bit R-2R DAC structure based on ordered element matching," Proc. IEEE Int. Symp. Circuits Syst. (2015) 1030 (DOI: 10.1109/ISCAS.2015.7168812).

[21] Y. Li, et al.: "A high resolution and high accuracy R-2R DAC based on ordered element matching," Proc. IEEE Int. Symp. Circuits Syst. (2013) 1974 (DOI: 10.1109/ISCAS.2013.6572256).

[22] I. Galton: "Why dynamic-element-matching DACs work," IEEE Trans. Circuits Syst. II, Exp. Briefs 57 (2010) 69 (DOI: 10.1109/ TCSII.2010.2042131).

[23] J. Liu, et al.: "A 14-bit 1.0-GS/s dynamic element matching DAC with $>80 \mathrm{~dB}$ SFDR up to the Nyquist," Proc. IEEE Int. Symp. Circuits Syst. (2015) 1026 (DOI: 10.1109/ISCAS.2015.7168811).

[24] B. Liu, et al.: "A novel dynamic element match technique in currentsteering DAC," IEEE 10th International Conference on ASIC (2013) 1 (DOI: 10.1109/ASICON.2013.6811898).

[25] R.A. Meaney and R.J. Speer: U.S. Patent 5075677 (1991).

[26] D. Marche, et al.: "An improved switch compensation technique for inverted ladder DACs," IEEE Trans. Circuits Syst. I, Reg. Papers 56 (2009) 1115 (DOI: 10.1109/TCSI.2008.2008510).

[27] D.S. Karadimas and K.A. Efstathiou: "On the study of a digitally calibrated R-2R ladder architecture," Int. Symp. Signals, Circuits Syst. (2005) 135 (DOI: 10.1109/ISSCS.2005.1509869).

[28] S.-C. Huang, et al.: "A wide range differential difference amplifier: a basic block for analog signal processing in MOS technology," IEEE Trans. Circuits Syst. II, Analog and Digital Signal Processing 40 (1993) 289 (DOI: 10.1109/82.227369).

[29] H. Alzaher and M. Ismail: "A CMOS fully balanced differential difference amplifier and its applications," IEEE Trans. Circuits Syst. II, Analog and Digital Signal Processing 48 (2001) 614 (DOI: 10.1109/82.943332).

[30] H. Fan, et al.: "A 4-channel 12-bit high-voltage radiation-hardened digital-to-analog converter for low orbit satellite applications," IEEE Trans. Circuits Syst. I, Reg. Papers 65 (2018) 3698 (DOI: 10.1109/ TCSI.2018.2856851).
[31] Texas Instruments, Inc.: "12-Bit quad voltage output digital-to-analog converter," Datasheet DAC7724 (1999). 